\title{
Reduction of the microbiological load on hospital surfaces through probiotic-based cleaning procedures: A new strategy to control nosocomial infections
}

\begin{abstract}
Traditional chemical disinfectants used in cleaning procedures in hospitals display several disadvantages such as limited in time biocide action, rapid bacterial re-contamination of treated surfaces, development of multidrug resistance by microorganisms (although so far demonstrated only in vitro) environmental pollution and potential onset of chemical sensitivity in patients, workforce and cleaners. According to recent experimental studies, cleaning techniques based on microbial bio-stabilization by using probiotic-based products are promising.

This study was aimed to evaluate the efficacy of a new procedure of sanitation with cleaning products containing spore forms of Bacillus spp, in comparison with a traditional chlorinebased treatment. Results from in vitro data, obtained under contamination-controlled conditions, prompted us to conduct an experimental study within two wards and outpatient departments in a study-model hospital. The total microbial count as well as Staphylococcus aureus, Coliforms, Pseudomonas spp., and Candida spp. titers were monitored for 4 months on several surfaces. A total of 11.223 microbiological samples were collected, both 7 and 24 hours after the scheduled cleaning procedures, setting the pre-cleaning microbial load as a control parameter. Our data showed that, differently from traditional chemical-based disinfectants, the effect of the probiotic-based product led to a significant reduction $(>80 \%)$ of the microbial load of Staphylococcus aureus, Coliforms, Pseudomonas spp. and Candida spp., with a stable effect over time. Data obtained in the present study show that probioticbased detergents significantly reduce the presence of pathogenic bacteria on contaminated surfaces, and support the hypothesis of a mechanism mediated by bio-stabilization of the microbial load.
\end{abstract}

Volume I Issue 5 - 2014

\author{
Alberta Vandini,' Alessia Frabetti,' Paola \\ Antonioli, ${ }^{2}$ Daniela Platano, ${ }^{3}$ Alessio \\ Branchini, ${ }^{4}$ Maria Teresa Camerada,' Luca \\ Lanzoni,' Piergiorgio Balboni,' Sante \\ Mazzacane' \\ 'CIAS Laboratory, Centre for the Study of Physical, Chemical \\ and Microbiological Contamination of Highly Sterile \\ Environments, Department of Architecture, University of \\ Ferrara, Italy \\ ${ }^{2}$ Infection Prevention Control and Risk Management \\ Department, Sant' Anna University Hospital of Ferrara, Italy \\ ${ }^{3}$ Department of Biomedical and Neuromotor Sciences, \\ University of Bologna, Italy \\ ${ }^{4}$ Department of Life Sciences and Biotechnology, University of \\ Ferrara, Italy
}

\begin{abstract}
Correspondence: Sante Mazzacane, CIAS Laboratory, Centre for the Study of Physical, Chemical and Microbiological Contamination of Highly Sterile Environments, Department of Architecture, University of Ferrara,Via Quartieri, 8, Ferrara, Italy, Tel 39-0532-293605, Email sante.mazzacane@unife.it
\end{abstract}

Received: July 04, 20I4 | Published: October 06, 2014

Keywords: surface contamination, probiotics, sanitation, healthcare-associated infections

Abbreviations: AU, out-patient department; CBS, chemical chlorine-based solution; CFU, colony-forming units; GM, general medicine department; PCHS, probiotic cleaning hygiene system, TMC, total microbial count

\section{Introduction}

Healthcare-Associated Infections (HAIs) are the most frequent complications occurring in healthcare facilities. It has been estimated that about $5 \%$ to $15 \%$ of all hospitalized patients develop a HAI during hospitalization. ${ }^{1}$ A three point-prevalence survey conducted in Italy showed a frequency of $6.7 \% \mathrm{HAI},{ }^{2}$ with the most frequent $\mathrm{HAI}$ represented by lower respiratory tract infections, followed by urinary tract infections. In 2012, the European Centre for Disease Prevention and Control (ECDC) published European report on HAI frequency, ${ }^{3}$ stating that every year over 3 million patients contract a HAI in the European Union. The high incidence of this severe complication is responsible for a huge cost on the society and makes the abatement of HAIs a priority in healthcare industry. A great number of studies and surveys based on the screening of patients have been reported, but the actual sources of contamination have been poorly characterized. In this view, the contribution of environment and surfaces to HAI development is still a matter of discussion. Nevertheless, it is well known that surfaces act as reservoirs for microorganisms, ${ }^{4}$ and represent a potential risk of cross-contamination for patients, caused by direct or indirect contact of patients with such potentially contaminated surfaces. To reduce these risks, in healthcare facilities sanitation procedures are applied to each surface that directly or indirectly may come in contact with people and patients. Despite the existence of experimental evidence suggesting that a reasonable, strategic use of disinfectants is recommended, the routine use of these agents remains still a matter of debate. ${ }^{5-7}$ Notwithstanding, a proper surface disinfection is highly recommended by all international guidelines as a primary procedure for preventing infections. ${ }^{8-10}$ However, the widespread application of chemical disinfectants may account for several risks both for the environment and for the safety of users. Since microorganisms can adapt to a variety of environmental physical and chemical conditions, it is therefore not surprising that acquired resistance to extensively used antiseptics and disinfectants, together with the ever increasing antimicrobial resistance, has been reported in vitro. ${ }^{11-14}$ For these reasons, there is increasing interest about the improvement of efficient, though sustainable, sanitation methodologies capable of containing or limiting the proliferation of pathogenic microorganisms. The importance of this issue is also outlined by recent research aimed to evaluate the antibacterial efficacy of natural detergents/biodetergents as a potential alternative 
to conventional disinfectants. ${ }^{15,16}$ A very promising strategy, as previously suggested by Falagas \& Makris, ${ }^{17}$ is represented by the use of non pathogenic microbiological products to colonize surfaces in order to counteract the proliferation of other bacterial species, in accordance with the competitive exclusion principle (Gause's law).$^{18}$ This approach is based on the well-established adoption of probiotics as food supplements, referred as live microorganisms which, when administered in adequate amounts, confer a health benefit on the host. The problem of hospital hygiene is thus completely reversed, since the aim of the procedure is no more represented by a general disinfection, which minimizes the presence of any kind of microorganisms on hospital surfaces, but to counteract the development of potentially pathogenic strains, instead tolerating the presence of microorganisms that are not harmful to humans.

The broadening of the probiotic concept towards the environment has been designated as biocontrol when the application is antagonistic towards a certain pathogen. ${ }^{19}$ This strategy has been already successfully applied for the abatement of Legionella in water systems, ${ }^{20}$ but there is no evidence about its effective application on surfaces by means of cleaning. Accordingly, such bio-stabilization through cleaning procedures may introduce a new conception of cleaning systems targeted to the establishment of a controlled and less harmful microbiota instead of a generic removal of microorganisms from the environment. The aim of this study was to provide an experimental evaluation of the efficacy of a probioticbased solution containing spore forms of Bacillus spp in comparison with traditional chlorine-based chemical disinfectants. As a studymodel environment we chose the Sant'Anna University Hospital of Ferrara (Italy). The microbiological screening was focused on the most common HAI-related microorganisms known to reside on surfaces, namely Staphylococcus aureus, Coliforms, Pseudomonas spp. and Candida spp.. The outcome should provide whether the strategy of bio-stabilization with a probiotic-based product could act as an effective and sustainable alternative to chemical disinfectants for treating inanimate surfaces, in particular those located in a nosocomial context.

\section{Materials and methods}

\section{Sanitation solutions}

The probiotic-based solution used contains $1 \%$ spores $(30 \mathrm{x}$ $106 \mathrm{CFU} / \mathrm{ml}$ ) of probiotic bacteria (Bacillus subtilis, Bacillus pumilus and Bacillus megaterium), added with ionic surfactants $(0.6 \%)$, anionic surfactants $(0.8 \%)$ and enzymes (amylase, $0.02 \%)$. The probiotic-based cleaning procedure was defined as PCHS (Probiotic Cleaning Hygiene System). This product is manufactured by Chrisal (Lommel, Belgium). The exact composition of the probiotic-based preparation is restricted. A common chemical chlorine-based solution (CBS) containing $0.65 \%$ sodium hypochlorite and $0.02 \%$ surfactants was used as control (Actichlor, Diversey S.p.A., Italy).

\section{Bacterial strains and culture media}

For the in vitro tests, Escherichia coli ATCC 10536 (Chrisope Technologies, Lake Charles, LA, USA), Staphylococcus aureus ATCC 25923 (Chrisope Technologies) and Pseudomonas aeruginosa ATCC 9027 (PBI International, Milan, Italy) strains were cultivated on MacConkey Agar (Merck Millipore, Darmstadt, Germany), BairdParker Agar (Merck Millipore) and Cetrimide agar (BD Diagnostic Systems, Franklin Lakes, New Jersey, USA) media, respectively. The same culture media were used to detect the presence of correspondent species of microorganisms in the samples. For the in situ trial, the Tryptic Soy Contact Agar (TSA, Merck Millipore) was used for the Total Microbial Count (TMC) together with the above reported media. The growth of all bacterial strains was obtained by incubation at $37^{\circ} \mathrm{C}$ for $18-24$ hours followed by 48 hours incubation at room temperature. The presence of Candida spp. was determined by the Sabouraud Dextrose Contact Agar added with chloramphenicol (Merck Millipore), by incubation at $25^{\circ} \mathrm{C}$ for $72-90$ hours, followed by 48 hours incubation at room temperature.

\section{Identification of the pathogenic strains}

The identification of pathogenic strains, initially identified through the Gram staining, was assessed by using the API 20 E (bioMérieux, Inc, Durham, NC, USA) or BBL Enterotube II (BD Diagnostic Systems) for Coliforms including Escherichia coli, the API Staph (20500 bioMérieux, Inc) for Staphylococcus aureus, the BBL Oxi/ Ferm Tube II (BD Diagnostic Systems) for Pseudomonas spp., and the API AUX C for Candida spp..

\section{Quantification of the microbial load}

Counting was performed by preparing $10_{-6}$ and $10_{-7}$ dilutions of the test suspension in diluents buffer. A sample of $1 \mathrm{ml}$ for each dilution was inoculated in duplicate using the pour plate technique. ${ }^{21}$ Each 1 $\mathrm{ml}$ sample was transferred into separate Petri dishes and a volume of $15 \mathrm{ml}$ of melted TSA, previously cooled to $45^{\circ} \mathrm{C}$, was added. The number of Colony-forming Units (CFU) was determined by colony counting after incubation of TSA plates at $37^{\circ} \mathrm{C}$ for $20-24 \mathrm{~h}$. A further incubation of plates for 20-24 hours was necessary. The numbers of $\mathrm{CFU} / \mathrm{ml}$ in the bacterial stock suspension were finally calculated.

\section{Sanitation procedures}

Sanitation procedures were performed using microfiber mops, cleaned after each use according to the manufacturer's instructions and colour-coded according to the type of target surface. A dry dust mopping phase was followed by a wet cleaning phase fresh prepared just before each use, with aqueous solutions of either the probioticbased or the chemical-based solutions. They were the test and control preparations, respectively. The microfiber cloths were soaked into the solution and stored inside clean containers until use. Mopping phases were performed always by the same trained operator, in order to exclude or minimize the introduction of potential variables in the implementation of the procedures. Floors were treated with $700 \mathrm{ppm} /$ $\mathrm{m} 2$ active chlorine. Hand/body-touched surfaces such as doorknobs, bed frames, tables and chairs or sink, toilet and other bathroom fixtures, were treated with $140 \mathrm{ppm} / \mathrm{m}^{2}$ active chlorine. These concentrations were used according to the manufacturer's specifications. Following the manufacturer's instructions (Chrisal), a preparation of $1.5 \mathrm{x}$ 106 spores $/ \mathrm{m} 2$ of the probiotic-based solution was used for the treatment of the same surfaces describe above. During the testing, the environments were used in the usual way, either by personal care, and patients and visitors.

\section{Contamination-controlled experiments (in-vitro tests)}

In-vitro experiments were conducted by treating samples of the hospital's areas current materials (i.e. ceramic, PVC, rubber, vitreouschina) with the probiotic-based solution. A solution containing a known concentration $\left(30 \times 10^{6}\right.$ cells $\left./ \mathrm{ml}, 15 \mathrm{ml} / \mathrm{m}^{2}\right)$ of Escherichia coli, Pseudomonas aeruginosa and Staphylococcus aureus strains was used for the contamination of the sampling surfaces. The bacterial load 
was measured by the determination of the colony count on RODAC plates (BD), containing TSA medium added with lecithin, histidine and Tween-20, in order to neutralize the action of disinfectants. The number of colonies was determined as total microbial count (TMC), or as specific colony count, by exploiting strain-specific medium. Control plates for sterility were used to guarantee sterility.

\section{In-situ trial}

The same sanitation procedure described for the contaminationcontrolled tests was applied for the in situ trial in two different hospital settings in the Sant'Anna University Hospital of Ferrara. They are referred to as in-patients general medicine setting (GM) and out-patient setting (AU) and in which the probiotic-based and chemical-based procedures were used alternatively. The GM setting consisted of the S-Area and T-Area sections (these are the original names of the two areas used since the construction of the hospital), and the Ophthalmology/Cardiology and Orthopedics departments were part of the AU setting. The experimental design for cleaning procedures, sampling surfaces and areas are summarized in Table 1. Evaluation of the probiotic-based sanitation procedure was performed for four months (March - August 2011), in three different time periods and. At the end of the first month, the sanitation protocols were inverted within the area/division of each setting. In order to verify the effectiveness and stability of the probiotic-based protocol, at the end of the second month of sampling collection, the study was prolonged for two more months in the GM setting areas. This choice was due both to the results of the trial, which remained constant in the last period for the PCHS protocol, and to the not negligible fact that over 11,000 microbiological samples had already been carried out, with very high costs accordingly. During the trial, neither the cleaning procedure (PCHS or CBS) nor the products used were never changed. These all belonged to the same purchased batch. The packaging of the probiotic product and the chemical product did not require special measures for their conservation, as indicated by the manufacturers. During the first and second periods, samplings were collected once a week at 07:00 a.m. (time point t1) and at 02:00 p.m. (time point t2), 30 minutes and 7 hours after sanitation, respectively. During the third period, samples were collected twice a week at 02:00 p.m. (time point t2), and at 06:00 a.m. of the day following the sanitation procedure (time point $\mathrm{t} 3$ ), immediately before the daily cleaning procedures. A total of 11,223 microbiological surface samples were processed following the microbiological environment monitoring methodology (MEM). ${ }^{22}$ Microbiological samplings were performed on two different floor locations in the same corridor (at the beginning and at the end of the corridor), randomly on one point on the toilet floor, and on the sink surface. In total, 33 different surfaces were sampled. Each measure was performed three times.

Table I Design of microbiological sampling and test conditions in the study-model hospital

\begin{tabular}{|c|c|c|c|c|c|}
\hline \multirow[b]{3}{*}{ Sampling } & \multirow[b]{3}{*}{ Type } & \multicolumn{4}{|c|}{ Sampling Areas } \\
\hline & & \multicolumn{2}{|l|}{$\mathbf{G M}^{\mathbf{a}}$} & \multicolumn{2}{|l|}{$A U^{b}$} \\
\hline & & T-Area & S-Area & O/C Dept ${ }^{c}$ & Orth Dept ${ }^{d}$ \\
\hline \multirow{3}{*}{ Sampling Surfaces } & Corridor floor (beginning and end) & ceramic & rubber & ceramic & rubber \\
\hline & Room toilet floor & ceramic & ceramic & ceramic & ceramic \\
\hline & Room toilet sink & vitreous-china & vitreous-china & vitreous-china & vitreous-china \\
\hline \multirow{3}{*}{ Sampling Conditions } & Ist period (I samplings/week) - I month & $\mathrm{PCHS}^{\mathrm{e}}$ & $\mathrm{CBS}^{f}$ & PCHS & CBS \\
\hline & $2^{\text {nd }}$ period (I samplings/week) - I month & CBS & PCHS & CBS & PCHS \\
\hline & $3^{\text {rd }}$ period (2 samplings/week) - 2 months & $\mathrm{PCHS}$ & PCHS & n.m. ${ }^{g}$ & n.m. \\
\hline
\end{tabular}

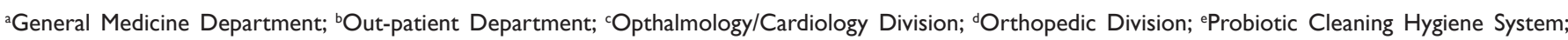
${ }^{f}$ Chemical Chlorine-based Solution; ${ }^{8}$ Not Monitored

\section{Calculation of the microbial load}

The pathogen reduction obtainable with the PCHS protocol was evaluated in percentage terms compared to the values obtained with the CBS protocol. The average value of each pathogen (measured in $\mathrm{CFU} / 100 \mathrm{~cm}^{2}$ ) obtainable with the CBS protocol in periods 1 and 2 (Table 1) was compared with that relating to the period 3, obtained with the probiotic product. For the first or second sampling periods, the calculation of the microbial load was performed by using the following formula:

$$
\text { percred }=\frac{C F U_{m i \quad C B S}-C F U_{m i} P R B}{C F U_{m i} C B S}
$$

With

$$
\begin{aligned}
& C F U_{m i \quad C B S}=\frac{\sum_{\tau} C F U_{i C B S}}{n} \\
& C F U_{m i} \quad P R B=\frac{\sum_{\tau} C F U_{i P R B}}{n}
\end{aligned}
$$

Where all the expressions are referred to: perc red: load

$\mathrm{CFU}_{\mathrm{miCBS}}: \quad$ Mean value of the microbial load of a specific pathogen, expressed as $\mathrm{CFU} / 100 \mathrm{~cm}^{2}$, calculated for the CBS protocol application

$\mathrm{CFU}_{\text {mi PCHS: }}$ Mean value of the microbial load of a specific pathogen, expressed as $\mathrm{CFU} / 100 \mathrm{~cm}^{2}$, calculated for the PCHS protocol application

$\mathrm{CFU}_{\mathrm{iCBS}}: \quad \mathrm{CFU} / 100 \mathrm{~cm}^{2}$ of a specific pathogen related to a specific sampling during the application of the CBS protocol

$\mathrm{CFU}_{\mathrm{i} \mathrm{PCHS}}: \quad \mathrm{CFU} / 100 \mathrm{~cm}^{2}$ of a specific pathogen related to a specific sampling during the application of the PCHS protocol

$$
\begin{array}{ll}
\tau: & \text { Summation extended to the sampling period } \\
\mathrm{n}: & \text { Number of samplings }
\end{array}
$$

Calculation of the microbial load for data collected during the third sampling period was performed only for the GM setting by using the formula: 


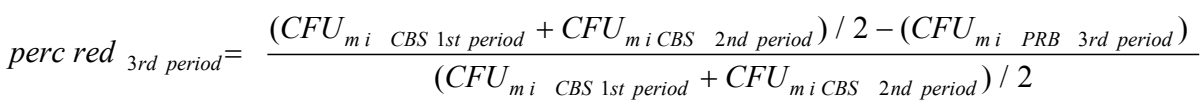

\section{Ethics statement}

The study protocol was reviewed and approved by the Ethics Committee, named Comitato Unico della Provincia di Ferrara (Unique Committee of the Ferrara Province), of the Azienda OspedalieroUniversitaria of Ferrara (Ferrara, Italy). The Ethics Committee stated that a formal authorization was not necessary because the probiotic products would not be directly administered to patients but exploited for cleaning of hospital surfaces only. For this reason, the Committee waived the need for written informed consent from participants because of the observational nature of the study.

\section{Statistical analysis}

All statistical analyses were performed using the SPSS software (SPSS Inc., Chicago, IL, United States). Normality was tested by the Wilk-Shapiro test. ${ }^{23}$ Differences among groups were tested by the non-parametric Mann-Whitney and Kruskall-Wallis tests. Results are indicated as mean \pm standard deviation. $\mathrm{P}$ values $\leq 0.05$ were considered as significant.

\section{Results}

Evaluation of the probiotic-based solution in contamination-controlled experimental settings

To investigate the pathogenic strain reduction capacity exerted by the probiotic-based solution, the efficacy under contaminationcontrolled conditions was analyzed in vitro. Selected surfaces, such as ceramic, PVC and rubber, were contaminated with known amounts of Escherichia coli, Staphylococcus aureus and Pseudomonas aeruginosa and then the bacterial load was monitored over time. Before treatment with the probiotic-based solution similar average $\mathrm{CFU} / \mathrm{cm}^{2}$ values among the three strains on the contaminated surfaces were observed. Interestingly, a reduction in the amount of pathogens on test surfaces was clearly evident one hour after treatment, with a significant lowering in the number of colonies within 30 minutes for all the strains tested. A barely detectable bacterial load was maintained up to 5 days of measurement, while unvarying bacterial loads were recognized on the untreated control surfaces (Table 2). These results indicate that the application of the probiotic-based product results in a significant lowering of specific bacterial loads in a contaminatedcontrolled context.

\section{In situ trial of the probiotic-based sanitation procedure}

In order to test the efficacy of this new cleaning procedure on a real context, the experimental evaluation was performed in a hospital environment. Sampling data, obtained in the hospital settings, are shown in Tables 3-5. Table 3 shows the comparison of the microbial load between the two hospital settings (GM e AU, see Table 1) during the first and second experimental periods, in which a reduction in the microbial load of the monitored pathogens was still evident.

Table 2 Time-course of average values of the bacterial load in contamination-controlled experiments in the absence (-) and after (+) the treatment with the probiotic-based solution

\begin{tabular}{|c|c|c|c|c|c|c|c|c|c|c|}
\hline \multirow[b]{3}{*}{ Bacterial Strain } & \multirow[b]{3}{*}{ Treatment } & \multicolumn{9}{|c|}{ Average bacterial load (CFU/cm²) } \\
\hline & & \multicolumn{4}{|c|}{ Time (Minutes) } & \multicolumn{5}{|c|}{ Time (Days) } \\
\hline & & 0 & 15 & 30 & 60 & I & 2 & 3 & 4 & 5 \\
\hline \multirow{2}{*}{ E. coli (ATCC I0536) } & - & $1.6 \times 10^{7}$ & $1.6 \times 10^{7}$ & $1.2 \times 10^{7}$ & $1.2 \times 10^{7}$ & $1.3 \times 10^{7}$ & $1.2 \times 10^{7}$ & $1.0 \times 10^{7}$ & $1.3 \times 10^{7}$ & $1.5 \times 10^{7}$ \\
\hline & + & $1.0 \times 10^{7}$ & $5.9 \times 10^{4}$ & $1.6 \times 10^{3}$ & $<10^{\mathrm{a}}$ & $<10^{\mathrm{a}}$ & $<10^{\mathrm{a}}$ & $<10^{\mathrm{a}}$ & $<10^{a}$ & $<10^{a}$ \\
\hline \multirow{2}{*}{ S. aureus (ATCC 25923) } & - & $1.0 \times 10^{7}$ & $1.0 \times 10^{7}$ & $1.0 \times 10^{7}$ & $1.0 \times 10^{7}$ & $1.0 \times 10^{7}$ & $1.0 \times 10^{7}$ & $1.0 \times 10^{7}$ & $9.0 \times 106$ & $7.8 \times 106$ \\
\hline & + & $2.5 \times 10^{7}$ & $1.6 \times 10^{4}$ & $1.6 \times 10^{4}$ & $<10^{\mathrm{a}}$ & $<10^{\mathrm{a}}$ & $<10^{\mathrm{a}}$ & $<10^{\mathrm{a}}$ & $<10^{\mathrm{a}}$ & $<10^{\mathrm{a}}$ \\
\hline \multirow{2}{*}{ P. aeruginosa (ATCC 9027) } & - & $1.4 \times 10^{7}$ & $1.6 \times 10^{7}$ & $1.7 \times 10^{7}$ & $1.9 \times 10^{7}$ & $1.9 \times 10^{7}$ & $1.9 \times 10^{7}$ & $1.7 \times 10^{7}$ & $1.7 \times 10^{7}$ & $1.4 \times 10^{7}$ \\
\hline & + & $1.8 \times 10^{7}$ & $5.0 \times 10^{7}$ & $6.0 \times 10^{3}$ & $<10^{\mathrm{a}}$ & $<10^{\mathrm{a}}$ & $<10^{a}$ & $<10^{\mathrm{a}}$ & $<10^{a}$ & $<10^{a}$ \\
\hline
\end{tabular}

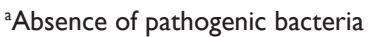

Table 3 Comparison of the average microbial load during the first and second experimental periods

\begin{tabular}{|c|c|c|c|c|c|c|c|c|}
\hline & \multicolumn{8}{|c|}{ CFU $/ 100 \mathrm{~cm}^{2}$} \\
\hline & \multicolumn{4}{|l|}{ GM } & \multicolumn{4}{|l|}{$\mathrm{AU}$} \\
\hline & \multicolumn{2}{|l|}{$\left.\right|^{\text {st }}$ period } & \multicolumn{2}{|c|}{$2^{\text {nd }}$ period } & \multicolumn{2}{|l|}{$\left.\right|^{\text {st }}$ period } & \multicolumn{2}{|l|}{$2^{\text {nd }}$ period } \\
\hline & Area $\mathbf{S}$ & Area T & Area S & Area T & O/C Dept & Orth Dept & O/C Dept & Orth Dept \\
\hline & CBS & PRB & PRB & CBS & CBS & PRB & PRB & CBS \\
\hline S. aureus & 1005 & 640 & 954 & 1354 & 911 & 689 & 914 & II4I \\
\hline Coliforms & 226 & 45 & 26 & 208 & 329 & 63 & 69 & 158 \\
\hline Pseudomonas spp. & 93 & 13 & 26 & 72 & 145 & 13 & 20 & 61 \\
\hline Candida spp. & 199 & 52 & 28 & 135 & 148 & 37 & 54 & 123 \\
\hline
\end{tabular}


Table 4 Average microbial load during the three scheduled experimental periods ( $P$ values were obtained by the Mann-Whitney analysis)

\begin{tabular}{|c|c|c|c|c|c|c|c|c|}
\hline \multirow[b]{2}{*}{ Procedure } & \multirow[b]{2}{*}{ Time Period } & \multicolumn{2}{|l|}{ Sampling ${ }^{a}$} & \multicolumn{5}{|c|}{ Average Bacterial Load (CFU/I00 cm²) } \\
\hline & & Time Point & $\mathbf{n}$ & $\mathrm{TMC}^{\mathrm{b}}$ & S. aureus & Coliforms & Pseudomonas spp. & Candida spp. \\
\hline & $\left.\right|^{\text {st }}-2^{\text {nd }}$ & $\mathrm{t}_{1}(07: 00 \text { a.m. })^{\mathrm{e}}$ & 7 & 301.76 & 49.2 & 12.02 & 5.53 & 4.78 \\
\hline \multirow[t]{3}{*}{ CBSc Protocol } & & $\mathrm{t}_{2}(02: 00 \text { p.m. })^{f}$ & 21 & 1055.05 & 110.96 & 23.05 & 9.17 & 15.02 \\
\hline & & & & $p=0.001$ & $p=0.001$ & $p=0.001$ & $p=0.001$ & $p=0.001$ \\
\hline & $1^{\text {st }}-2^{\text {nd }}$ & $\mathrm{t}_{1}(07: 00 \text { a.m. })^{\mathrm{e}}$ & 7 & 1072.05 & 58.86 & 5 & 1.8 & 6.08 \\
\hline \multirow[t]{3}{*}{$\mathrm{PCHS}^{d}$ Protocol } & & $\mathrm{t}_{2}(02: 00 \text { p.m. })^{f}$ & 21 & 1033.62 & 81.65 & 3.67 & 0.76 & 4.26 \\
\hline & & & & $p=0.024$ & $p=0.001$ & $p=0.007$ & $p=0.067$ & $p=0.325$ \\
\hline & $3^{\text {rd }}$ & $\mathrm{t}_{3}(06: 00 \text { a.m. })^{\mathrm{g}}$ & 7 & 622.81 & 26.23 & 12 & 4.82 & 12.12 \\
\hline \multirow[t]{2}{*}{ PCHS Protocol } & & $\mathrm{t}_{2}(02: 00$ p.m. $)$ & 10 & 632.34 & $12.8 \mid$ & 5 & 1.32 & 3.82 \\
\hline & & & & $p=0.001$ & $p=0.024$ & $p=0.030$ & $p=0.007$ & $p=0.003$ \\
\hline
\end{tabular}

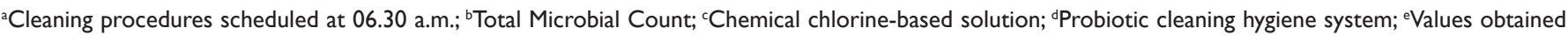
$1 / 2$ hour after the cleaning procedures; ${ }^{f}$ Values obtained $61 / 2$ hour after the cleaning procedures; ${ }^{8}$ Values obtained 24 hours after the cleaning procedures and before the new sanitation operation.

Table 5 Effects of the probiotic-based sanitation solution on the microbial load in comparison with the chemical-based treatment during the Ist and 2 nd periods

\begin{tabular}{lllllll}
\hline \multicolumn{7}{c}{ Average Bacterial Load $\left(\mathrm{CFU} / \mathbf{1 0 0} \mathbf{c m}^{2}\right)$} \\
\hline Time Point & Protocol & TMC $^{\mathbf{2}}$ & S. aureus & Coliforms & Pseudomonas spp. & Candida spp. \\
\hline \multirow{4}{*}{$\mathrm{t}_{1}$} & CBS & 301.76 & 49.2 & 12.02 & 5.53 & 4.78 \\
& PCHS & 1072.05 & 58.86 & 5 & 1.8 & 6.08 \\
& & $p=0.001$ & $p=0.014$ & $p=0.001$ & $p=0.005$ & $p=0.666$ \\
$\mathrm{t}_{2}$ & CBS & 1055.05 & 110.96 & 23.05 & 9.17 & 15.02 \\
& PCHS & 1033.62 & 81.65 & 3.67 & 0.76 & 4.26 \\
& & $p=0.180$ & $p=0.001$ & $p=0.001$ & $p=0.001$ & $p=0.001$ \\
\hline
\end{tabular}

${ }^{a}$ Total Microbial Count

Mean values for the GM and AU hospital settings are not separately reported in Table 4 because no statistically significant differences in the microbiological load were found, despite the different type of health care activity. The PCHS and the CBS cleaning procedures have always been performed at 06:30 a.m.. As already mentioned, the microbiological load was monitored in three stages: half an hour after the application of the cleaning procedures (t1, 7 a.m.), about 6 $1 / 2$ hours later (t2, 2 p.m.) and about 24 hours later (t3, 6 a.m. of the next day and before the new cleaning). The $\mathrm{t} 3$ instant corresponds to the time when there was the maximum value of contamination of the surfaces. The mean TMC values observed at $\mathrm{t} 1$ with the probioticbased solution were significantly higher than those obtained with the chemical-based product, due to the presence on treated surfaces of non-pathogenic microorganisms of the probiotic-based solution itself. Indeed, the probiotic microorganisms accounted for about $98 \%$ of TMC. The comparison of the two different protocols showed statistically significant differences in the microbial loads at time point t1. From Table 4 it can be observed that, for the CBS procedure, the $S$. aureus value was equal to $49.2 \mathrm{CFU} / 100 \mathrm{~cm}^{2}$ at $\mathrm{t} 1$ time (half an hour after cleaning), but it becomes $110.96 \mathrm{CFU} / 100 \mathrm{~cm} 2$ at $\mathrm{t} 2$ time (nearly 7 hours after cleaning). That is to say its value doubles after about 7 hours. A very similar trend is noted for Coliforms, Pseudomans spp and Candida spp..

On the contrary, with regard to the PCHS procedure, the value of $S$. aureus was equal to 58.86 and $81.65 \mathrm{CFU} / 100 \mathrm{~cm}^{2}$ at $\mathrm{t} 1$ time and at 2 time, respectively. For other pathogens, the value even decreased as time goes by, probably because they are more sensitive to the action of the PCHS protocol's Bacillus spp., which continue to exert their competitive action for a long time even after their application (unlike chemical disinfectants, whose action expires quickly). This comparison is summarized in Table 5. Returning to Table 4, it is interesting to note the data obtained in the $3 \mathrm{rd}$ period. When the PCHS procedure was applied for several weeks (as for the S-Area, Table 1 ), the average values of the pathogens measured at $t 2$ time further decreased and reached lower values than those for the CBS protocol: for the $S$. aureus 12,81 against $110.96 \mathrm{CFU} / 100 \mathrm{~cm}^{2}$, for Coliforms 5.0 against $23.05 \mathrm{CFU} / 100 \mathrm{~cm}^{2}$, for Pseudomonas spp. 1.32 against $9.17 \mathrm{CFU} / 100 \mathrm{~cm}^{2}$, and for Candida spp. 3.82 against $15.02 \mathrm{CFU} / 100$ $\mathrm{cm}^{2}$. Even more interesting is to observe what happened after almost 24 hours after cleaning. In this case, at t1 time of the 3 rd period $S$. aureus reached $26.23 \mathrm{CFU} / 100 \mathrm{~cm}^{2}$ with the PCHS protocol, against $110.96 \mathrm{CFU} / 100 \mathrm{~cm}^{2}$ at $\mathrm{t} 2$ time with the CBS protocol. The trend is the same for all pathogens considered. This means that the values achieved by the pathogen 24 hours after the cleaning with the PCHS protocol were well below the values reached after only $61 / 2$ hours using the CBS protocol. It is therefore quite evident the effect of containment of pathogen levels in the interval of time between one cleaning procedure and the other, when using the product based on probiotics. The treatment with the probiotic-based product reduced the potentially pathogenic microbial load, with the exception of the TMC and the Staphylococcus aureus and Candida spp. titers, while 
an increase was observed after the application of the chemical-based product. Conversely, as well as for other pathogens tested, a reduction in the microbial load of both Staphylococcus aureus and Candida spp. was observed at $\mathrm{t} 2$ (Table 5).

These results indicated a statistically significant general reduction of the potentially pathogenic species after the treatment with the probiotic-based product.

\section{Microbiological stabilization effect of the probiotic- based procedure}

In order to verify the effectiveness and stability of the probioticbased procedure, the study was prolonged for two more months within the GM setting areas (see Methods and Table 4). Noticeably, when the bacterial load, collected at $\mathrm{t} 2$ in the first and second period of the survey, was compared with the microbial load obtained in the third period, always at $\mathrm{t} 2$ time, a significant reduction was obtained for all average bacterial loads, with the only exception of Coliforms $(p=0.485)$ (Table 6). These results suggested that the prolonged use of the probiotic-based protocol significantly improved the level of sanitation of treated surfaces, with a stabilization of the microbial load over time, with constant microbiological contamination values over time. This phenomenon was very evident in researches subsequently conducted; these results will be further described in other future works.

Table 6 Stabilization effect of the probiotic-based sanitation procedur

\begin{tabular}{lllllll}
\hline \multicolumn{7}{c}{ Average Bacterial Load $\left(\mathrm{CFU} / 100 \mathrm{~cm}^{2}\right)$} \\
\hline Time Point & Time Period & TMC & S. aureus & Coliforms & Psudomonas spp. & Candida spp. \\
\hline $\mathrm{t}_{2}$ & $1^{\mathrm{st}}-2^{\text {nd }}$ & 1033.62 & 81.65 & 3.67 & 0.76 & 4.26 \\
& $3^{\text {rd }}$ & 632.34 & 12.81 & 5 & 1.32 & 3.82 \\
& & $p=0.001$ & $p=0.001$ & $p=0.485$ & $p=0.008$ & $p=0.003$ \\
\hline
\end{tabular}

${ }^{2}$ Total Microbial Count

The percentage reduction of the bacterial loads for different surfaces, sampled at $\mathrm{t} 2$, confirmed that the probiotic-based procedure resulted in a general reduction (up to $80 \%$ ) and stabilization of the pathogenic load (Table 7). In addition, data obtained in the third period, during which sampling was performed 24 hours later the sanitation procedure with the probiotic-based protocol, showed that the average microbial loads were lower than those obtained at $\mathrm{t} 2$, by using the chemical-based protocol. Overall, these data indicate that, differently from observations obtained with the chemical-based product, the probiotic-based protocol resulted in an efficient stabilization of the microbial load, which was maintained at low levels within the 24 hours until the subsequent sanitation procedure.

Table 7 Percentage reduction of pathogen strains per sampling surface and sampling areas during the three experimental periods, after the application of the probiotic-based sanitation procedure

\begin{tabular}{|c|c|c|c|c|c|}
\hline \multirow[b]{2}{*}{ Pathogen } & \multicolumn{5}{|c|}{ Sampling Surfaces } \\
\hline & Sampling Area & Time Period & Corridor & Room Toilet Floor & Room Toilet Sink \\
\hline \multirow{3}{*}{ Staphylococcus aureus } & $\mathrm{AU}$ & $I^{\text {st }}$ & $12.16 \%$ & $58.75 \%$ & $55.74 \%$ \\
\hline & GM & $2^{\text {nd }}$ & $28.31 \%$ & $51.33 \%$ & $52.50 \%$ \\
\hline & & $3^{\text {rd }}$ & $81.03 \%$ & $85.88 \%$ & $95.59 \%$ \\
\hline \multirow{3}{*}{ Coliforms } & $\mathrm{AU}$ & $\left.\right|^{\text {st }}$ & $82.09 \%$ & $89.15 \%$ & $81.56 \%$ \\
\hline & GM & $2^{\text {nd }}$ & $50.29 \%$ & $78.13 \%$ & $75.83 \%$ \\
\hline & & $3^{\text {rd }}$ & $79.72 \%$ & $78.31 \%$ & $85.12 \%$ \\
\hline \multirow{3}{*}{ Pseudomonas spp. } & $\mathrm{AU}$ & $I^{\text {st }}$ & $97.62 \%$ & $55.28 \%$ & $67.53 \%$ \\
\hline & GM & $2^{\text {nd }}$ & $42.24 \%$ & $75.94 \%$ & $50.41 \%$ \\
\hline & & $3^{\text {rd }}$ & $88.44 \%$ & $78.57 \%$ & $95.16 \%$ \\
\hline \multirow{3}{*}{ Candida spp. } & $\mathrm{AU}$ & $I^{\text {st }}$ & $77.54 \%$ & $82.90 \%$ & $50.38 \%$ \\
\hline & GM & $2^{\text {nd }}$ & $67.67 \%$ & $67.80 \%$ & $27.93 \%$ \\
\hline & & $3^{\text {rd }}$ & $68.47 \%$ & $71.78 \%$ & $94.86 \%$ \\
\hline
\end{tabular}

\section{Discussion}

The present study investigated the effectiveness of a probioticbased sanitation solution for hard surfaces both in a laboratory contamination-controlled setup and in a real setting, consisting of a study-model hospital. This study was based on the hypothesis that probiotic bacteria, which are defined as a preparation of viable microorganisms that bring a benefit to the host health, ${ }^{24}$ could colonize surfaces and counteract the proliferation of other bacterial species, ${ }^{17}$ including those recognized as potential pathogens for humans. Probiotics have been extensively used per os as food supplements for alterations, in both the qualitative and quantitative composition of human intestinal flora. Bacillus spp have been exploited as probiotics for a series of different applications, such as dietary supplementation 
in humans and animal feed, due to their capacity of stimulating the immune system. ${ }^{25}$ In particular, Bacillus subtilis has been indicated as safe for human consumption,,$^{26,27}$ in live and spore forms ${ }^{28}$ and there is increasing evidence and interest in the use of this non-pathogenic microorganism in the preparation of food. ${ }^{29}$

The promising strategy, based on probiotics, could be shifted from the feed supplement purpose to a new application in which these bacteria could be involved in the colonization of inanimate surfaces in order to minimize and contrast the proliferation of other bacterial strains, potentially pathogenic to human subjects. In this context, a probiotic-based sanitation procedure for hard surfaces can be hypothesized to have a role in the control of HAIs, which are the most frequent complications in healthcare facilities, ${ }^{1}$ and often develop from either direct or indirect contact with pathogen-contaminated surfaces.

The evidence for a proof-of-principle application of this strategy, stemmed from the results obtained in contamination-controlled studies, in which commercially available pathogenic strains such as Escherichia coli, Pseudomonas aeruginosa and Staphylococcus aureus were exploited. These experimental settings revealed that the probiotic-based solution resulted in a significant reduction in the pathogen populations used as reference, pointing towards the potential feasibility of this method on a real setting. To address the issue in a real context, the Sant'Anna University Hospital of Ferrara as a study-model setting was chosen. The observations of the probiotic-based solution indicate that its effectiveness in reducing and maintaining a low pathogen load was significantly more pronounced than that of a chemical-based disinfectant on all tested surfaces, over time. The reduction of pathogen load, observed using the probioticbased treatment, could be likely due to bio-stabilization, according to the competitive exclusion principle (Gause's law), ${ }^{18,30}$ and with other mechanisms related to natural antimicrobial activities, as demonstrated by several in-vitro studies ${ }^{31-33}$ and by the recent isolation and description of a Bacillus strain, displaying a broad-spectrum biofilm inhibition activity. ${ }^{34}$

We are aware of some limitations in this study, concerning the actual mechanisms and dynamics involved, which are beyond the purpose of the study itself. Noticeably, as mentioned above, one of the most well-known effects of probiotics is related to competitive exclusion, ${ }^{35}$ which confers an advantage to a certain bacterial strain on others, competing for the same resources. Nevertheless, several other mechanisms may contribute to this effect, such as quorum sensing/ quenching, ${ }^{36}$ or the production of multifunctional molecules, known as bio-surfactants, which have antagonistic anti-adhesive properties against microbial pathogens. ${ }^{37,38}$ Among these, one of the most representative is surfactin, produced by Bacillus subtilis, which is a surface-active lipopeptide belonging to the extracellular polymeric substances (EPS) ${ }^{39}$ displaying a potent antimicrobial activity. ${ }^{40}$ In addition, other competition-related processes such as cannibalism and predation are involved in the formation of the biofilm and in the antimicrobial activity of Bacillus subtilis, respectively. ${ }^{41-44}$

The proposed probiotic-based treatment, at variance with traditional chemical-based sanitation procedures, gradually reduced microbial contamination, with a consolidation of its effect in 8-12 weeks since the first application. Present data indicate that the probiotic-based procedure exerted a continuous activity, either by affecting the nutritional sources available for the development of microorganisms or, more likely, by forming anti-pathogen biofilms on inanimate surfaces. When a traditional chemical-based disinfectant was used, the potential pathogenic microbial load had a steep increase within 7 hours, resulting in a doubling or tripling of the number of $\mathrm{CFU} / \mathrm{cm}^{2}$. Conversely, the increase of microbial load after the application of the probiotic-based solution was modest within the next 24 hours. This observation suggests that the probiotic-based solution displayed a long-term stabilizing effect that strongly lowered the risk of peaks in pathogen loads between consecutive applications, which represents one of the most deleterious hurdles frequently witnessed for disinfections with chemical-based solutions.

This study provides indications on the need for a careful use of the surface microbiological sampling through the TMC monitoring, for the evaluation of environmental contaminations, because of the wide range of the microbial load, in particular when chemical disinfectants are used. Surface sampling for the evaluation of the disinfectant efficacy should be performed within 30 minutes after application, but it does not represent a suitable method to describe the daily "average" contamination of surfaces. Differently, in the case of the probioticbased treatment, the $\mathrm{CFU} / \mathrm{cm}^{2}$ count, targeted on a specific bacterial strain, provides a scientific value that is stronger than that yielded by the TMC, since the daily variation of the microbial load is modest.

A matter of debate could pertain the use of bacteria for sanitation procedures, notwithstanding the final goal of sanitation itself is not strictly the lowering of microorganisms in general, but rather the control or limitation of potentially pathogenic strain development, tolerating the presence of microorganisms harmless for humans. Indeed, regarding the risk related to the use of probiotic agents in indoor (or clinical) environments, the strict selection of strains must be of primary importance. It is worth noting that many probiotic strains are considered safe for use in humans, ${ }^{26,45,46}$ and that the Food and Drug Administration (FDA) classifies them as generally recognized as safe (GRAS) microorganisms. In addition, Bacillus subtilis has been listed on the Qualified Presumption of Safety (QPS) by the European Food Safety Authority since 2007. ${ }^{47,48}$ Nevertheless, we are aware that safety assessments, including antibiotic resistance and transferability, genetic stability, toxicity, remain important issues, still debated even for probiotics already designated for human consumption both in healthy and in sick individuals. ${ }^{49}$

For these reasons, when working in healthcare areas, hosting immune-compromised patients, the need and availability of proper solid data about safety and toxicity of the strain used, should be the absolute priority. On the other hand, the major pitfall of current chemical disinfection procedures and agents is related to the regrowth/re-contamination leading to unstable bacterial loads, thus potentially exposing patients residing in hospital rooms, to several HAI-related complications. Noticeably, this picture prepares the ground for the notion that the replacement of such pathogenic strains with probiotics, displaying a safer reputation, should result in a lower risk for patients to develop HAIs.

The present study demonstrated that the strategy of probioticbased sanitation results in an efficient and long-term lowering of pathogens both in contamination-controlled and hospital contexts. Our data indicated that i) a probiotic-based sanitation procedure was significantly more effective (up to $80 \%$ ) in reducing potentially pathogenic microbial loads than a traditional chlorine-based chemical disinfectant, and ii) the reduced microbial load was stably maintained at low levels throughout the 24 hours after application, despite of the presence of continuous and multiple sources of microbial recontaminations due to external natural contributors such as patients, visitors, hospital staff and moving materials. 
However, further studies are needed in order to identify new standards for hygiene monitoring, and to better understand the mechanisms underlying this probiotic sanitation technique. Environmental probiotics alone may not be adequate to control all microbial species and, in addition, routine chemical cleaning procedures may interfere with probiotics. ${ }^{15}$ Furthermore, it is an open challenge to determine whether, how and to what extent the strategy of probiotic-based sanitation of hard surfaces in hospital contexts will correlate with the number of HAIs.

\section{Conclusion}

In conclusion, this proposed strategy, based on the new PCHS system, provides evidence for the proof-of-principle of the strategy of bio-stabilization, and for probiotic-based products as a reliable alternative to traditional chemical disinfection of nosocomial surfaces, in particular of those directly or indirectly in contact with patients. However, this method is not proposed for environments that require a very low value of total microbial count (TMC), such as operating rooms, infectious disease wards, clean room, etc. In the future, the authors aspire to correlate the efficacy of this probioticbased procedure with the reduction of nosocomial infections.

Finally, in order to evaluate the susceptibility or resistance of the Bacillus strains to antibiotics, the study was implemented by exploiting antibiogram tests on Bacillus spp. colonies, coming from the same type of the sample surfaces (e.g. floor) monitored in the in situ trial. The first results indicate that the isolated Bacillus spp. strains are susceptible to all the antibiotics tested, with the exception of penicillin, towards which the Bacillus sp is naturally resistant, as also indicated by antibiograms performed on the commercially-available ATCC strain. These preliminary data are not presented here but will be part of the future research work, where also new results, stemming from molecular assays (i.e. qPCR) for the evaluation of resistance factors, will be described.

\section{Acknowledgments}

The authors would like to thank COPMA Company scrl for funding this study, and the boards of directors and the Ethics Committee of the hospital involved in this study.

\section{Conflicts of interest}

Authors declare that there is no conflict of interest.

\section{References}

1. Siegel JD, Rhinehart E, Jackson M, et al. 2007 Guideline for isolation precautions: preventing transmission of infectious agents in health care settings. Am J Infect Control. 2007;35(10 Suppl 2):S65-S164.

2. Lanini S, Jarvis WR, Nicastri E, et al. Healthcare-associated infection in Italy: annual point-prevalence surveys, 2002-2004. Infect Control Hosp Epidemiol. 2009;30(7):659-665.

3. European Centre for Disease Prevention and Control (ECDC). Poin prevalence survey of healthcare-associated infections and antimicrobial use in European acute care hospitals 2011-2012; 2013.

4. Hota B. Contamination, disinfection, and cross-colonization: are hospital surfaces reservoirs for nosocomial infection? Clin Infect Dis. 2004;39(8):1182-1189.

5. Rutala WA, Weber DJ. The benefits of surface disinfection. Am J Infect Control. 2005;33(7):434-435.

6. Dettenkofer M, Spencer RC. Importance of environmental decontamination-a critical view. J Hosp Infect. 2007;65(Suppl 2):55-57.
7. Daschner FD, Schuster A, Dettenkofer M, et al. No routine surface disinfection. Am J Infect Control. 2004;32(8):513-515.

8. Rutala WA. APIC guideline for selection and use of disinfectants. 1994, 1995, and 1996 APIC Guidelines Committee. Association for Professionals in Infection Control and Epidemiology, Inc. Am J Infect Control. 1996;24(4):313-342.

9. Sehulster L, Chinn RY, CDC, et al. Guidelines for environmental infection control in health-care facilities. Recommendations of CDC and the Healthcare Infection Control Practices Advisory Committee (HICPAC). MMWR Recomm Rep. 2003;52(RR-10):1-42.

10. Mangram AJ, Horan TC, Pearson ML, et al. Guideline for Prevention of Surgical Site Infection, 1999. Centers for Disease Control and Prevention (CDC) Hospital Infection Control Practices Advisory Committee. Am J Infect Control. 1999;27(2):97-132.

11. Frabetti A, Vandini A, Balboni P, et al. Experimental evaluation of the efficacy of sanitation procedures in operating rooms. Am J Infect Control. 2009;37(8):658-664

12. McDonnell G, Russell AD. Antiseptics and disinfectants: activity, action, and resistance. Clin Microbiol Rev. 1999;12(1):147-179.

13. Aiello AE, Larson E. Antibacterial cleaning and hygiene products as an emerging risk factor for antibiotic resistance in the community. Lancet Infect Dis. 2003;3(8):501-506

14. Russell AD. Biocide use and antibiotic resistance: the relevance of laboratory findings to clinical and environmental situations. Lancet Infect Dis. 2003;3(12):794-803.

15. Liguori G, Bagattini M, Galle F, et al. Microbiological evaluation of the efficacy of two new biodetergents on multidrug-resistant nosocomial pathogens. Ann Clin Microbiol Antimicrob. 2009;8:35.

16. Liguori G, Bagattini M, Galle F, et al. Automated cleaning of fan coil units with a natural detergent-disinfectant product. Ann Clin Microbiol Antimicrob. 2010;9:29.

17. Falagas ME, Makris GC. Probiotic bacteria and biosurfactants for nosocomial infection control: a hypothesis. J Hosp Infect. 2009;71(4):301-306.

18. Hardin G. The competitive exclusion principle. Science. 1960;131(3409):1292-1297.

19. Gatesoupe FJ. The use of probiotics in aquaculture. Aquaculture. 1999;180(1-2):147-165.

20. Temmerman R, Vervaeren H, Noseda B, et al. Inhibition of Legionella pneumophila by Bacillus sp. Eng Life Sci. 2007;7(5):497-503.

21. Hoben HJ, Somasegaran P. Comparison of the pour, spread, and drop plate methods for enumeration of Rhizobium spp. in inoculants made from presterilized peat. Appl Environ Microbiol. 1982;44(5):1246-1247.

22. Pitzurra M, Savino A, Pasquarella C. Microbiological environment monitoring (MEM). Ann Ig. 1997;9(6):439-454.

23. Hirano SS, Nordheim EV, Arny DC, et al. Lognormal distribution of epiphytic bacterial populations on leaf surfaces. Appl Environ Microbiol. 1982;44(3):695-700.

24. Schrezenmeir J, de Vrese M. Probiotics, prebiotics, and synbioticsapproaching a definition. Am J Clin Nutr. 2001;73(2 Suppl):361S-364S.

25. Duc le H, Hong HA, Fairweather N, et al. Bacterial spores as vaccine vehicles. Infect Immun. 2003;71(5):2810-2818.

26. Sorokulova IB, Pinchuk IV, Denayrolles M, et al. The safety of two Bacillusprobiotic strains for human use. Dig Dis Sci. 2008;53(4):954-963.

27. Duc le H, Hong HA, Barbosa TM, et al. Characterization of Bacillus probiotics available for human use. Appl Environ Microbiol. 2004;70(4):2161-2171. 
28. Hong HA, Duc le H, Cutting SM. The use of bacterial spore formers as probiotics. FEMS Microbiol Rev. 2005;29(4):813-835.

29. Hong HA, Huang JM, Khaneja R, et al. The safety of Bacillus subtilis and Bacillus indicus as food probiotics. J Appl Microbiol. 2008;105(2):510-520.

30. La Ragione RM, Woodward MJ. Competitive exclusion by Bacillus subtilis spores of Salmonella enterica serotype Enteritidis and Clostridium perfringens in young chickens. Vet Microbiol. 2003;94(3):245-256.

31. Balcazar JL, Rojas-Luna T. Inhibitory activity of probiotic Bacillus subtilis UTM 126 against vibrio species confers protection against vibriosis in juvenile shrimp (Litopenaeus vannamei). Curr Microbiol. 2007;55(5):409-412.

32. Ravi AV, Musthafa KS, Jegathammbal G, et al. Screening and evaluation of probiotics as a biocontrol agent against pathogenic Vibrios in marine aquaculture. Lett Appl Microbiol. 2007;45(2):219-223.

33. Nakayama T, Lu H, Nomura N. Inhibitory effects of Bacillus probionts on growth and toxin production of Vibrio harveyi pathogens of shrimp. Lett Appl Microbiol. 2009;49(6):679-684.

34. Wu Z, Ye C, Guo F, et al. Evidence for broad-spectrum biofilm inhibition by the bacterium Bacillus sp. strain SW9. Appl Environ Microbiol. 2013;79(5):1735-1738.

35. Mountzouris KC, Balaskas C, Xanthakos I, et al. Effects of a multispecies probiotic on biomarkers of competitive exclusion efficacy in broilers challenged with Salmonella enteritidis. Br Poult Sci. 2009;50(4):467-478

36. Zhu J, Kaufmann GF. Quo vadis quorum quenching? Curr Opin Pharmacol. 2013;13(5):688-698.

37. Servin AL. Antagonistic activities of lactobacilli and bifidobacteria against microbial pathogens. FEMS Microbiol Rev. 2004;28(4):405-440.

38. Rivardo F, Turner RJ, Allegrone G, et al. Anti-adhesion activity of two biosurfactants produced by Bacillus spp. prevents biofilm formation of human bacterial pathogens. Appl Microbiol Biotechnol. 2009;83(3):541-553.
39. Flemming HC, Neu TR, Wozniak DJ. The EPS matrix: the "house of biofilm cells". J Bacteriol. 2007;189(22):7945-7947.

40. Gonzalez DJ, Haste NM, Hollands A, et al. Microbial competition between Bacillus subtilis and Staphylococcus aureus monitored by imaging mass spectrometry. Microbiology. 2011;157(Pt 9):2485-2492.

41. Gonzalez-Pastor JE, Hobbs EC, Losick R. Cannibalism by sporulating bacteria. Science. 2003;301(5632):510-513.

42. Gonzalez-Pastor JE. Cannibalism: a social behavior in sporulating Bacillus subtilis. FEMS Microbiol Rev. 2011;35(3):415-424.

43. Lopez D, Vlamakis H, Losick R, et al. Cannibalism enhances biofilm development in Bacillus subtilis. Mol Microbiol. 2009;74(3):609-618.

44. Nandy SK, Bapat PM, Venkatesh KV. Sporulating bacteria prefers predation to cannibalism in mixed cultures. FEBS Lett. 2007;581(1):151-156.

45. Adams MR, Marteau P. On the safety of lactic acid bacteria from food Int J Food Microbiol. 1995;27(2-3):263-264.

46. Reid G. Probiotics and prebiotics- progress and challenges. International Dairy Journal. 2008;18(10-11):969-975.

47. European Food and Safety Authority. Scientific opinion on the maintenance of the list of QPS biological agents intentionally added to food and feed (2013 update). EFSA J. 2013;11(11):34-49.

48. Scientific Committee of the European Food and Safety Authority. Introduction of a qualified presumption of safety (QPS) approach for assessment of selected microorganisms referred to EFSA. Opinion of the Scientific Committee. EFSA J. 2007;587:1-16.

49. Sanders ME, Akkermans LM, Haller D, et al. Safety assessment of probiotics for human use. Gut Microbes. 2010;1(3) 